\title{
PRODUCCIÓN Y TRANSPORTE DE SEDIMENTO EN CÁRCAVAS DESARROLLADAS EN LA DEPRESIÓN INTERIOR ALTOARAGONESA (CUENCA DE ARAGUÁS, PIRINEO CENTRAL)
}

\author{
D. REGÜÉS ${ }^{(1)}$, E. NADAL-ROMERO(1) J. LATRON $^{(2)}$ y C. MARTÍ-BONO ${ }^{(1)}$ \\ (1) Instituto Pirenaico de Ecología (CSIC), \\ Campus de Aula Dei, Avenida Montañana 1005, 50192 Zaragoza, España. \\ (2) Institut de Diagnosi Ambiental i Estudis de l'Aigua (IDAEA-CSIC), \\ c/ Sole i Sabarís, s/n. 08028 Barcelona. \\ correo electrónico: dregues@ipe.csic.es
}

\begin{abstract}
RESUMEN. Este estudio muestra el análisis de la dinámica del sedimento en suspensión procedente de las cárcavas desarrolladas en la Depresión Interior Altoaragonesa (Pirineo Central). La presencia de estas morfologías destaca por su frecuencia y por su impacto sobre las redes de drenaje, debido a su intensa dinámica geomorfológica. La formación y desarrollo de estas morfologías está asociada a dos factores principalmente: las características geológicas del substrato, constituido por margas, y el contraste estacional que rige el clima en la zona, definido como submediterráneo de montaña. El estudio geomorfológico de estas morfologías se inició en 2004, a partir de seleccionar y equipar una pequeña cuenca, mediante parcelas y dispositivos para el seguimiento de los procesos de meteorización y erosión (desde enero de 2004 hasta diciembre de 2006). La monitorización de la cuenca se completó con la instalación de una estación de aforo (octubre de 2005) en el barranco de la Rebullesa, que facilita el seguimiento de la respuesta hidrológica y del transporte de sedimento en suspensión. En este trabajo se presenta un análisis basado en 79 eventos registrados entre octubre de 2005 y abril de 2007. Los resultados, a pesar de corresponder a un periodo de tiempo reducido, han permitido destacar algunas de las características generales de la respuesta hidro-sedimentológica propias de estas morfologías: una elevada producción de escorrentía, incluso en los momentos de gran sequedad; una gran rapidez e intensidad en la respuesta hidrológica; una enorme capacidad de producción y exportación de sedimento, con concentraciones que puntualmente pueden alcanzar más de $1000 \mathrm{~g} \cdot \mathrm{l}^{-1}$, y la fuerte sensibilidad que demuestran las cárcavas frente a la estacionalidad climática. Asimismo, se ha constatado que la intensidad de precipitación muestra una buena correlación con la concentración de sedimento, especialmente durante los periodos más secos, lo cual confirmaría
\end{abstract}


que el principal proceso de generación de escorrentía en las cárcavas es la superación de la capacidad de infiltración (flujos Hortonianos).

ABSTRACT. This study shows the analyses of suspended sediment transport from badland areas developed in the Inner Depression (Central Pyrenees). The importance of these morphologies is related to its frequency, and to the high impact that they produce on the drainage net, because of their intense geomorphological dynamics. The occurrence and development of these morphologies is associated to two main factors: the geological characteristics of the substratum constituted by marls, and the strong climatic seasonality of the submediterranean climate. The area has been studied since 2004, through the selection of a small basin, which was monitored with sensors and plots for observation of weathering and erosion processes (between January 2004 and December 2006). This instrumentation was completed with a gauging station (October 2005) on the main drainage (Rebullesa ravine) that facilitates the record of hydrological response and suspended sediment transport. This paper shows an analysis, based on 79 recorded events between October 2005 and April 2007. The results, that correspond to a short period of time, have allowed pointing out some general characteristics of hydro-sedimentological responses in these morphologies: very high hydrological response, even during the driest period; great capacity of sediment yield and exportation, with punctual concentrations than can reach more than $1000 \mathrm{~g} \cdot \mathrm{l}^{-1}$, and the strong sensibility against climatic seasonality. Likewise, it was observed that precipitation intensity is well correlated with sediment concentration, especially during the driest periods, which demonstrates that the main runoff generation process is associated to infiltration excess mechanisms (Hortonian flow).

Palabras clave: cárcavas, respuesta hidrológica, concentración de sedimento, transporte en suspensión.

Key words: badlands, hydrological response, sediment concentration, suspended transport.

Enviado el 15 de septiembre de 2008 Aceptado el 12 de diciembre de 2008

\section{Introducción}

La presencia de paisajes acarcavados suele asociarse habitualmente a regiones semiáridas, debido al fuerte impacto paisajístico que producen por el gran desarrollo y extensión que llegan a alcanzar. Un claro ejemplo de ello se puede encontrar en el sureste de la Península Ibérica (López-Bermúdez y Romero-Díaz, 1989; Calvo y Harvey, 1996; Solé-Benet et al., 1997). Sin embargo, durante las últimas décadas se ha evidenciado la presencia de cárcavas en una amplio espectro de ambientes y condiciones climáticas (Gallart et al., 2002). Este rango de posibilidades incluyen zonas de montaña donde las precipitaciones pueden ser relativamente abundantes y, por tanto, no cabría esperar que la vegetación sufriera algún tipo de limitación. En este sentido, estudios específicos 
sobre procesos de meteorización en cárcavas del Pirineo Oriental han permitido identificar y evaluar algunas de las causas que explican esta situación contradictoria (Regüés et al., 2000b) y comprender mejor los mecanismos que regulan su funcionamiento hidrológico y los procesos de erosión (Regüés y Gallart, 2004). Así, se ha constatado que la dinámica geomorfológica de las cárcavas de montaña está muy afectada por los procesos de meteorización física (Antoine et al., 1995; Regüés et al., 1995; Nadal-Romero et al., 2007) motivando que la intensidad con que actúan los procesos de generación de escorrentía y erosión muestre variaciones temporales que pueden ser bastante notables.

Por otro lado, los estudios de carácter hidro-sedimentológico en cárcavas han revelado similitudes que pueden ser generalizables a todas estas morfologías, con independencia del ambiente y la litología en que se han formado. Así, se puede atribuir a todas las cárcavas una elevada capacidad de respuesta hidrológica y producción de sedimento (Yair et al., 1980; Bryan y Yair, 1982; Cerdà y Navarro, 1997; Torri y Rodolfi, 2000; Cantón et al., 2001; Regüés et al., 2000a; Mathys et al., 2005; Nadal-Romero et al., 2008a y 2008b). Estas características implican que la presencia de este tipo de morfologías causa un impacto significativo en las redes de drenaje de la zona, mediante la incorporación súbita de caudales y un gran aporte de sedimento. Un estudio sobre la identificación de áreas fuente de sedimento en la Depresión Interior Altoaragonesa (Pirineo Central), mediante la aplicación de técnicas de teledetección, SIG y modelos hidrológicos (Beguería, 2005), ha identificado los afloramientos de litologías margosas como las principales áreas contributivas. Asimismo, en la mencionada Depresión Altoaragonesa, se han identificado más de un centenar de morfologías acarcavadas desarrolladas sobre el substrato margoso (Nadal-Romero et al., 2006b) y que constituyen una densa red de cuencas y barrancos tributarios del río Aragón, ocupando una extensión de unos $15 \mathrm{~km}^{2}$ y representando un $3,4 \%$ de la superficie total de la Depresión.

Durante los últimos veinte años han proliferado de manera significativa los estudios sobre transporte de sedimento en suspensión en cuencas del área mediterránea. Esto se debe, por un lado, a que algunos estudios concluyen que el transporte de sedimento en suspensión constituye el mecanismo dominante en la citada región (Webb et al., 1995) y, por otro lado, a la posibilidad de utilizar sistemas de medición en continuo que facilitan el estudio de cuencas mediante su monitorización (Alexandrov et al., 2003). Los estudios realizados en cárcavas de montaña situadas en el área mediterránea han revelado que el volumen de sedimento producido por estas morfologías alcanza valores extremadamente elevados (Regüés et al., 2000a; Mathys et al., 2005) que pueden superar en más de un orden de magnitud las tasas específicas estimadas en campos agrícolas o en cárcavas de ambientes más áridos. Sin embargo, la información disponible al respecto es todavía escasa y dispersa; por ello es necesario profundizar en este tipo de estudios, lo cual puede ser de gran utilidad a la hora de evaluar el impacto que causaría una eventual proliferación de estas morfologías e incluso las consecuencias derivadas del cambio climático.

Este estudio propone como principal objetivo el análisis y evaluación de los procesos que intervienen en la producción y exportación de sedimento en suspensión en una cárcava situada en la Depresión Interior Altoaragonesa, con la finalidad de: i) describir 
la dinámica espacio-temporal de producción de sedimento; ii) evaluar la magnitud aproximada que puede alcanzar la tasa media anual de transporte; iii) cuantificar la magnitud del impacto sedimentológico que produce el conjunto de estas morfologías en las redes fluviales.

\section{2. Área de estudio}

La Depresión Interior Altoaragonesa se encuentra situada en la vertiente sur del Pirineo Central, formando un corredor elevado (con una altitud media de unos $550 \mathrm{~m}$ s.n.m.), con una longitud aproximada de $80 \mathrm{~km}$ y una anchura media de unos $6,5 \mathrm{~km}$. La depresión presenta una orientación oeste-este y se encuentra encajada entre relieves más elevados, constituidos por turbiditas de la facies Flysch al norte y por Molasas (areniscas, arcillas y margas) al sur, mientras la depresión se encuentra sobre un substrato constituido por margas gris-azuladas masivas (Margas de Larrés) con estratos esporádicos y delgados de turbiditas. También destaca la presencia ocasional de núcleos de concreciones carbonatadas interestratificadas de dimensiones y morfologías variables y más resistentes a la erosión que las margas (Martí-Bono y Plana, 1996). Asimismo, el paisaje está caracterizado por la presencia de más de un centenar de morfologías acarcavadas a lo largo de toda la Depresión, cuyo desarrollo está asociado al tipo de substrato. Además, debido a la gran frecuencia con que se encuentran y su distribución, su formación podría estar relacionada a cambios de usos del suelo y otras modificaciones derivadas de explotaciones forestales y agrícolas. El análisis mineralógico de estas margas indicó que están compuestas básicamente por minerales arcillosos (44\% de illita y clorita), calcita y dolomita $(41 \%)$ y cuarzo $(15 \%)$.

La zona seleccionada para este estudio es una pequeña cuenca $\left(0,45 \mathrm{~km}^{2}\right)$ que se caracteriza por presentar una densa red de cárcavas en su parte inferior. La cuenca se encuentra en la parte central de la vertiente norte de la Depresión Altoaragonesa, a unos $9 \mathrm{~km}$ hacia el noroeste de Jaca (Fig. 1) y junto a la localidad de Araguás del Solano, por

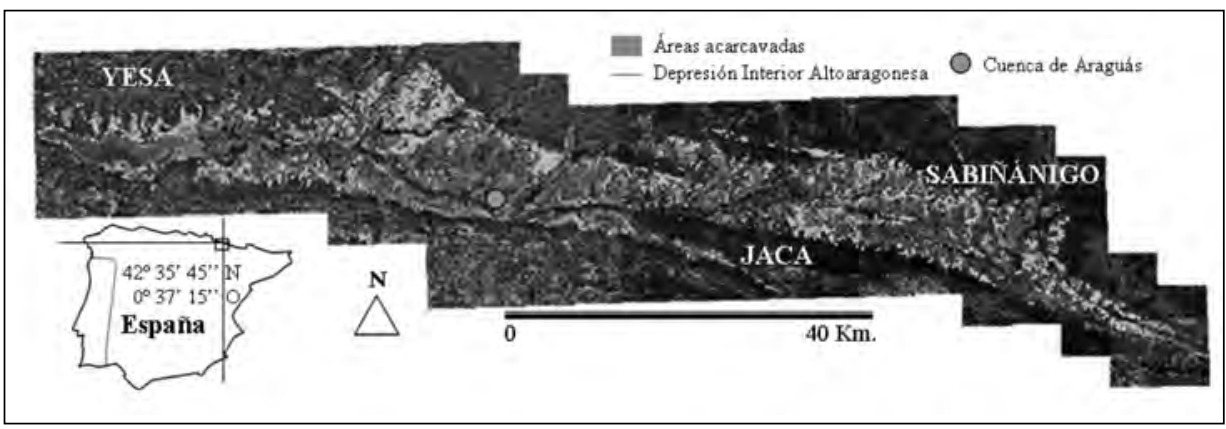

Figura 1. Localización de la cuenca de Araguás en el marco de la Depresión Interior Altoaragonesa 
este motivo ha sido identificada como cuenca de Araguás, aunque el barranco que la constituye se conoce como de "La Rebullesa". El punto más elevado de la cuenca ronda los 1100 m s.n.m. y el más bajo se sitúa alrededor de los 780 m s.n.m. La cuenca presenta las típicas pendientes pronunciadas que caracterizan los paisajes abarrancados o acarcavados, con un valor medio próximo al $20 \%$, aunque las pendientes más pronunciadas se encuentran en las laderas norte de las cárcavas, dónde son habituales los taludes casi verticales. En este sentido, es importante destacar la presencia de tres áreas claramente diferenciadas, asociadas a distintos usos del suelo:

1. La parte superior forestal (30\% de la cuenca): destaca la importante presencia de restos de muros de antiguas terrazas de cultivo. A partir de los años 50 se produce el abandono de los cultivos y la zona fue reforestada con Pinus sylvestris durante los años 60-70.

2. La parte intermedia de prados ( $45 \%$ de la cuenca): es la zona con menores pendientes de la cuenca, en ella destacan los pastos dedicados a caballos y ovejas principalmente, dónde antiguamente hubieron campos de cultivo. Este sector esta parcialmente ocupado por matorral de recolonización espontánea.

3. La parte baja de cárcavas ( $25 \%$ de la superficie): es el sector que presenta pendientes más pronunciadas, debido a la presencia de una densa red de cárcavas muy desarrolladas y encajadas. Su formación podría estar relacionada a la deforestación y los cambios de usos del suelo. Esta zona presenta esporádicas manchas de matorral con algún pino poco desarrollado, especialmente en los fondos del barranco y en algunos sectores de las laderas orientadas hacia el sur. En este caso, el fuerte ritmo de la dinámica geomorfológica impide que la vegetación se pueda asentar en las laderas de las cárcavas. Se observan frecuentemente movimientos en masa, desprendimientos y deslizamientos de fragmentos del suelo, que proceden del límite superior de los barrancos o de pequeñas plataformas que todavía se conservan en los fondos.

El clima de la zona ha sido definido como submediterráneo de montaña o de transición, porque presenta la característica distribución estacional de las precipitaciones y de las temperaturas del clima mediterráneo, pero recibe influencia atlántica, que se manifiesta mediante la entrada de masas de aire húmedas y frescas (Creus, 1983). La temperatura media anual se sitúa en torno a los $10^{\circ} \mathrm{C}$ y muestra un contraste estacional muy acentuado (con mínimas que alcanzan habitualmente los $-14^{\circ} \mathrm{C}$ y máximas que rondan $\operatorname{los} 35^{\circ} \mathrm{C}$ ); asimismo, se producen fuertes oscilaciones térmicas diarias que pueden superar $\operatorname{los} 20^{\circ} \mathrm{C}$. Entre mediados de otoño y la primera mitad de la primavera se producen heladas habitualmente, que afectan especialmente a las vertientes umbrías. La precipitación media anual se sitúa en torno a $1.000 \mathrm{~mm}$, con una variabilidad interanual de unos $200 \mathrm{~mm}$, y se registra una media de 85 días de lluvia al año, concentrándose en otoño y primavera, aunque también son frecuentes las tormentas convectivas en verano y las precipitaciones en forma de nieve durante el invierno (Beguería y Vicente-Serrano, 2006). 


\section{Dispositivo experimental}

La cuenca de Araguás esta monitorizada, desde finales del año 2005, mediante una estación de aforo que se encuentra en el límite de la zona acarcavada, que corresponde aproximadamente al punto en que el torrente de La Rebullesa abandona la cuenca de Araguás, a unos $500 \mathrm{~m}$ de su confluencia con el río Lubierre (afluente del río Aragón). La estación de aforo esta formada por un canal principal que tiene una amplitud de 2,1 $\mathrm{m}$, y con un pequeño canal de estiaje $(0,30 \mathrm{~m}$ de profundidad por $0,50 \mathrm{~m}$ de anchura) que permite estimar los caudales menores con mayor precisión (Figura 2). La estación de aforo esta equipada con los siguientes instrumentos:

- Medición de caudales- Sensor de ultrasonidos (Pepperl+Fuchs) que proporciona la altura del nivel de agua cuando supera $l o s 30 \mathrm{~cm}$ y una sonda de presión (Keller DCX-22) instalada en el canal de estiaje para registrar los caudales más bajos, aunque su rango de detección permite registrar todas las alturas de la lámina de agua.

- Medición del transporte de sedimento en suspensión- Turbidímetro de IR (Endress+Hauser) para la estimación de la concentración de sedimento en suspensión a partir del registro en continuo de la turbidez. El rango de detección de este instrumento, obtenido mediante calibración con sedimento procedente del lecho del torrente (Nadal-Romero, 2008), se extiende entre valores de $0,1 \mathrm{~g} \cdot \mathrm{l}^{-1} \mathrm{y}$ de $2.500 \mathrm{~g} \cdot \mathrm{l}^{-1}$.

- Medición del transporte de sedimento en disolución- Muestreador automático (ISCO 3700) equipado con 24 botellas de medio litro y activado en función de las oscilaciones del nivel de agua y de turbidez, lo cual garantiza la adquisición de muestras puntuales durante las crecidas.

Por otro lado la cuenca está equipada mediante tres pluviómetros de balancín (Davis Instruments) que registran las precipitaciones en tres puntos de la cuenca, dos de ellos en la zona de cárcavas (780 y 800 m s.n.m.) y el tercero próximo al límite inferior de la zona forestal (1.000 m s.n.m.). El rango de detección de estos pluviómetros es de 0,2mm.

Asimismo, se instalaron unos sistemas muy sencillos para evaluar cualitativamente las características texturales del material transportado en suspensión. Estas trampas de sedimento consistieron en dos tubos de PVC de 1 metro de longitud y $4 \mathrm{~cm}$ de diámetro interior en los que se cerró uno de los extremos con una red mosquitera fina $(0,5 \mathrm{~mm}$ de luz) para retener el sedimento atrapado en su interior y permitir la salida del agua. Estas trampas se instalaron en el centro del lecho torrencial, enterrando casi todo el tubo por el extremo cerrado con un ángulo bajo, quedando la parte abierta del tubo situada a 5 y $12 \mathrm{~cm}$ de altura sobre la superficie del lecho. De esta manera, el extremo de cada tubo quedó situado en dirección opuesta al flujo del caudal y dispuesto para ser rellenado de sedimento cuando el nivel del agua lo cubriera. Estos dispositivos se instalaron en dos ocasiones, verano de 2006 e invierno de 2007, con la finalidad de obtener información en dos situaciones bien diferenciadas. 


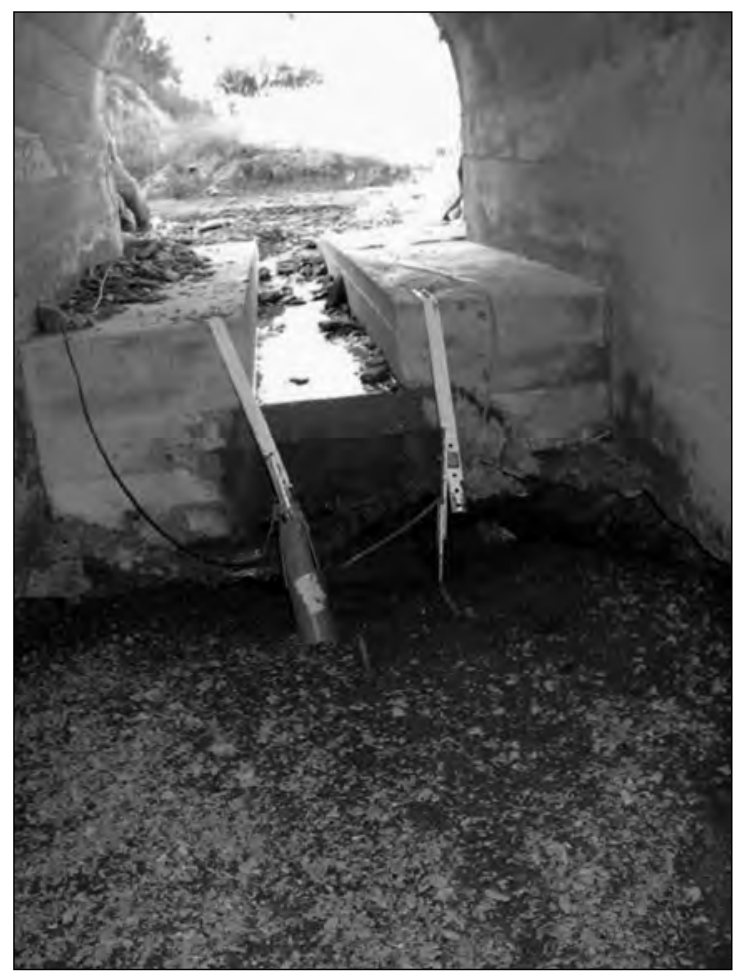

Figura 2. Detalle de la estación de aforo instalada en la cuenca de Araguás

\section{Análisis de los resultados}

Es importante destacar que el estudio e interpretación del transporte de sedimento en suspensión en estas cárcavas requiere un conocimiento previo de su funcionamiento geomorfológico, con respecto a la secuencia espacio-temporal que rige las interacciones entre los procesos de meteorización y erosión, y también es importante conocer las características básicas de la respuesta hidrológica, tanto de las cárcavas como de otros ambientes presentes en la zona de estudio.

\subsection{Dinámica de la producción de sedimento}

El estudio geomorfológico de las cárcavas desarrolladas en la Depresión Interior Altoaragonesa se inició en enero de 2004, mediante la selección y monitorización de parcelas para el estudio de los procesos de meteorización y de erosión que afectan a estas morfologías. Los resultados obtenidos a partir de estos trabajos permitieron definir las pautas espaciales y temporales que determinan la dinámica del regolito superficial y su relación con la producción de sedimento en las laderas de estas cárcavas. Esta informa- 
ción ha permitido describir la secuencia de procesos que actúan desde que el regolito alcanza su máximo estado de degradación, quedando predispuesto para ser erosionado, hasta que el sedimento procedente de las laderas se acumula al pie de las vertientes en forma de abanicos, que son altamente susceptibles a ser movilizados y transportados hacia las redes de drenaje principales.

Con respecto a los procesos de meteorización física y química, su estudio demostró que la intensidad de estos procesos está muy relacionada con la orientación de las vertientes, siendo más activos y eficaces en las laderas umbrías. En este sentido, se ha constatado que la alteración de la marga está controlada por las condiciones térmicas e hídricas y, por tanto, se asocian a las variaciones climáticas estacionales (Nadal-Romero et al., 2006a y 2007). Así, el proceso de meteorización física más activo y eficaz está relacionado con los ciclos de hielo-deshielo, mientras la meteorización química está asociada a la disolución de carbonatos. En consecuencia, la meteorización de las margas se produce básicamente entre finales del otoño y principios de la primavera, cuando las condiciones ambientales son favorables para el crecimiento de cristales de hielo; como consecuencia de ello la marga pierde cohesión, muestra un aspecto muy frágil y blando y su estructura se ve afectada por un fuerte incremento de la porosidad, desarrollando una morfología tipo "popcorn" (Pardini et al., 1996) que predomina especialmente en las laderas con orientación norte. Durante los periodos más cálidos y secos la superficie de las cárcavas evoluciona hasta adquirir la morfología de una costra superficial, en cuyo caso presenta un aspecto compactado, duro y agrietado.

Por otro lado, el estudio de los procesos de erosión demostró que la acción de la salpicadura o "splash" está condicionada al estado físico que presenta el regolito superficial, siendo más eficiente cuanto más desarrollado o alterado se encuentre. Así, la mayor fragilidad de las margas se produce entre el final del invierno y el inicio de la primavera, coincidiendo con el mayor desarrollo de la morfología tipo "popcorn"; mientras el momento de mayor resistencia se produce entre finales del verano y principios del otoño, cuando la superficie se encuentra encostrada (Nadal-Romero, 2008; NadalRomero y Regüés, 2009). En este sentido, se debe mencionar que las condiciones físicas del regolito también influyen sobre la capacidad de generación de escorrentía y, por tanto, la transferencia del sedimento desde las laderas hacia las redes también presenta variaciones de carácter espacio-temporal. Un resultado similar se observó en cárcavas desarrolladas en el Pirineo Oriental, dónde se confirmó que el estado físico del regolito superficial es un factor determinante en la generación de escorrentía superficial y la transferencia de sedimento (Regüés y Gallart, 2004).

En síntesis, la dinámica temporal de la producción de sedimento en las laderas se puede simplificar considerando los momentos de mayor trascendencia:

i) Máxima alteración del regolito superficial: hacia finales del invierno, cuando la superficie presenta una morfología tipo "popcorn" que alcanza su mayor desarrollo en las vertientes con exposición norte. 
ii) Máxima susceptibilidad a la erosión por salpicadura: durante los primeros meses de la primavera, cuando las laderas todavía conservan una morfología tipo "popcorn" pero están afectadas por una rápida pérdida de humedad. En esta situación las laderas quedan muy expuestas a la erosión por salpicadura, pero su capacidad de generar escorrentía superficial es bastante reducida. Por este motivo, el efecto erosivo del impacto de las gotas de lluvia se manifiesta en el desarrollo de una costra superficial (McIntyre, 1958; Regüés y Torri, 2002) mientras la transferencia de sedimento hacia las redes de drenaje se incrementa de manera progresiva, a medida que se va desarrollando la costra superficial. Esto se pone de manifiesto mediante la progresiva formación de abanicos de acumulación al pie de las vertientes.

iii) Erosión por arroyada concentrada en las laderas: esta situación predomina entre finales de primavera y las primeras semanas de otoño. En esta fase el regolito superficial presenta una costra compacta y dura, favorecida por una fuerte desecación que causa la formación de grandes grietas de retracción. Las tormentas de verano, de alta intensidad pero corta duración, superan con facilidad la capacidad de infiltración en la superficie encostrada que muestran las cárcavas y se genera con cierta rapidez un flujo de escorrentía muy intenso, que se concentra frecuentemente siguiendo algunas de las grietas de retracción (Haigh, 1978). Esto queda evidenciado por la aparición de una densa red de regueros, a lo largo de las laderas y dispuestos paralelamente a la pendiente.

iv) Erosión por arroyada difusa en las laderas: este proceso de erosión es el predominante durante los primeros meses de otoño, mientras la superficie de las cárcavas presenta un regolito poco desarrollado. El estado físico característico del regolito en este periodo está definido por unas condiciones térmicas e hídricas favorables para mantener su superficie sellada, esto favorece que la respuesta hidrológica se produzca de manera casi inmediata y homogénea. La acción de la arroyada dispersa afecta a las laderas eliminando progresivamente el rastro de los regueros, también se observa la desaparición de los abanicos acumulados al pie de las vertientes.

\subsection{La respuesta hidrológica en las áreas acarcavadas}

En general, se ha observado que estas cárcavas muestran una gran capacidad de respuesta hidrológica, en comparación a la observada en áreas próximas con distintos usos del suelo y cubierta vegetal (Regüés et al., 2006; García-Ruiz et al., 2008). Esta característica ha sido confirmada por los volúmenes e intensidades de precipitación mínimos $\left(0,8 \mathrm{~mm}\right.$ y $1,2 \mathrm{~mm} \cdot \mathrm{h}^{-1}$ respectivamente) capaz de generar escorrentía de crecida (NadalRomero et al., 2008a).

También se ha podido constatar, a partir de regresiones lineales, que el pico de caudal está relacionado con el volumen e intensidad de precipitación $(\mathrm{R}=0,637$ y $\mathrm{R}=0,629$ respectivamente, siendo estadísticamente significativas al nivel 0,01 y n=79). Sin embargo, los resultados de este ajuste cambian significativamente si diferenciamos entre las 45 crecidas registradas durante periodos húmedos, en cuyo caso disminuye el coeficiente de 
correlación con respecto a la precipitación total y el ajuste con la intensidad de precipitación pierde toda significación estadística ( $\mathrm{R}=0,560$ y $\mathrm{R}=0,261$ respectivamente). Pero si consideramos las 34 crecidas registradas en las estaciones más secas, tanto la cantidad total de precipitación como la intensidad de precipitación mantienen la misma significación estadística ( $R=0,785$ y $R=0,668$, respectivamente). Estos resultados indican que durante las estaciones secas la respuesta hidrológica se produce exclusivamente en las zonas acarcavadas (Nadal Romero et al., 2008a) donde la generación de escorrentía se debe a la superación de la capacidad de infiltración (flujo Hortoniano), siendo la intensidad de precipitación uno de los factores más determinantes.

Como síntesis de las características generales de la respuesta hidrológica se pueden diferenciar dos situaciones asociadas a la distribución climática estacional:

i) un periodo húmedo (octubre-abril) en el que la respuesta hidrológica afecta tanto a las cárcavas como a las áreas con cubierta vegetal. En esta situación los caudales y los coeficientes de escorrentía alcanzan valores importantes y se combinan dos mecanismos de generación de escorrentía: por un lado una escorrentía superficial o subsuperficial por saturación del suelo en las áreas vegetadas y, por otro lado, una escorrentía superficial por superación de la capacidad de infiltración en las áreas acarcavadas.

ii) un periodo seco (mayo-septiembre) en el que la respuesta hidrológica generalmente está limitada a las zonas acarcavadas, siendo la escorrentía superficial por superación de la capacidad de infiltración el único mecanismo de generación de escorrentía y las cárcavas las únicas zonas contributivas. Esto queda evidenciado en una reducción de la magnitud media de los caudales y en unos coeficientes de escorrentía bastante más bajos, en comparación con los registros obtenidos durante el periodo húmedo.

\subsection{El transporte de sedimento en suspensión}

El estudio del transporte de sedimento en suspensión se ha realizado a partir de las 79 crecidas registradas que, a pesar de corresponder a un periodo de tiempo reducido (octubre de 2005-abril de 2007), comprenden una variedad de registros suficientemente extensa para poder analizar la información obtenida a escala de evento y obtener algunas conclusiones interesantes, aunque se deban limitar a los procesos que actúan en estas morfologías. Sin embargo, este análisis debe ser considerado como preliminar, puesto que no permite estudiar la variabilidad del proceso en una escala temporal más amplia. En cualquier caso, se ha estimado la tasa anual de exportación de sedimento con el propósito de adquirir una idea de su magnitud y tener algunas indicaciones de su repercusión en el contexto de toda la Depresión Interior. Esta información ha permitido también establecer comparaciones con los valores estimados en otras áreas acarcavadas y en ambientes menos afectados por la erosión. Asimismo, se ha realizado un análisis acumulando los datos mensualmente y estacionalmente, para poder relacionar la masa de sedimento transportado con distintos volúmenes de precipitación y escorrentía. 


\subsubsection{Dinámica del sedimento en suspensión}

Se ha analizado la granulometría del sedimento en suspensión a partir de cuatro muestras obtenidas mediante las trampas instaladas en el lecho torrencial. Estas muestras corresponden a dos crecidas (18/08/06 y 09/02/07) representativas de los periodos seco y húmedo (Tabla 1). La caracterización granulométrica (Fig. 3) de estas muestras ha proporcionado algunas indicaciones sobre el tipo de material transportado en suspensión. Así, se ha podido constatar que el tamaño de las partículas transportadas en suspensión disminuye en sentido vertical, puesto que en las muestras recogidas a $5 \mathrm{~cm}$ del lecho se han medido partículas de hasta $30 \mathrm{~mm}$ de diámetro, mientras a $12 \mathrm{~cm}$ del lecho las partículas mayores solo alcanzaron los10 mm de diámetro. Asimismo, estas muestras indican que durante la crecida de mayor magnitud, con un pico de caudal y de concentración más elevado (Tabla 1), también se incrementó el tamaño de las partículas transportadas en suspensión (Fig. 3). Estos resul-

Tabla 1. Características de las crecidas en las que fueron tomadas muestras de sedimento en suspensión mediante trampas situadas a dos alturas sobre el lecho del barranco de la Rebullesa

\begin{tabular}{|c|c|c|c|c|c|c|}
\hline Crecida & $\begin{array}{l}\text { Prec. Total } \\
\quad(\mathrm{mm})\end{array}$ & $\begin{array}{c}\text { Int. Máx. } \\
(5 \mathrm{~min})\left(\mathrm{mm} \mathrm{h}^{-1}\right)\end{array}$ & $\begin{array}{l}\text { Q Máx. } \\
\left(\mathbf{l ~ s}^{-1} \mathbf{k m}^{-2}\right)\end{array}$ & $\mathbf{C E}$ & $\begin{array}{l}\text { CSS Máx. } \\
\qquad\left(\mathrm{g} \mathrm{l}^{-1}\right)\end{array}$ & $\begin{array}{l}\text { Sed Total } \\
\text { (ton) }\end{array}$ \\
\hline $18-08-06$ & 8,8 & 33,6 & 458,5 & 0,06 & 145,3 & 31,05 \\
\hline 09-02-07 & 14,2 & 12 & 717,7 & 0,28 & 633,5 & 447,36 \\
\hline
\end{tabular}

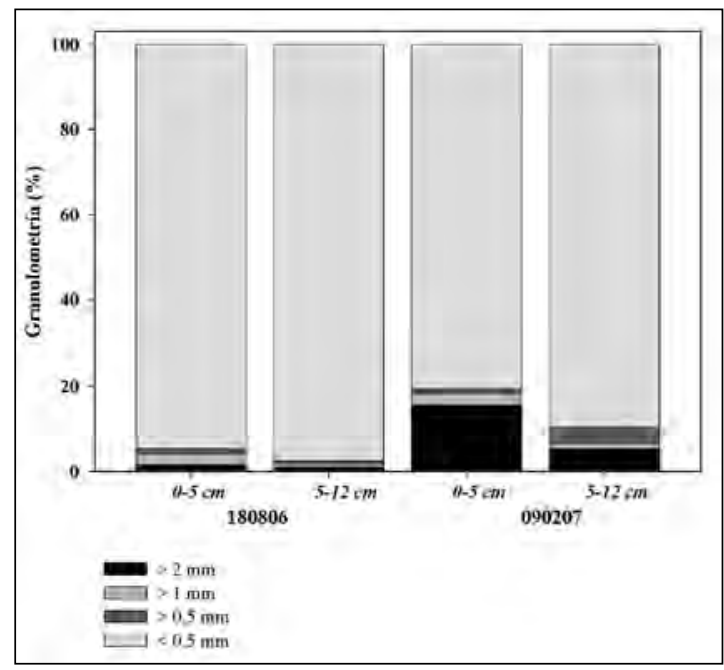

Figura 3. Distribución granulométrica de las partículas transportadas en suspensión durante dos eventos en la cuenca de Araguás. Las muestras fueron tomadas a 5 y $12 \mathrm{~cm}$ sobre el lecho del torrente de La Rebullesa 
tados coinciden con otros estudios desarrollados en ambientes de montaña media con cárcavas, dónde se ha constatado que el diámetro medio de las partículas transportadas en suspensión aumenta con el incremento del caudal y de la concentración (Soler y Gallart, 2006).

Esta información indica que el rango y variabilidad de las partículas transportadas en suspensión es bastante amplio en este tipo de ambiente, llegando incluso al nivel de las gravas de diámetro medio, y también que las características del material transportado en suspensión pueden ser muy heterogéneas, con diferencias significativas en su distribución vertical. Todo esto implica una mayor dificultad en la estimación de la carga sólida en suspensión a partir del registro de turbidez, especialmente si tenemos en cuenta que la atenuación de la transmisividad del medio es inversamente proporcional al diámetro de las partículas. Este efecto es mayor cuando las partículas son mayores de 100 um, según Campbell y Spinrad (1987), de manera que las partículas de mayor tamaño se pueden considerar como "invisibles" para los turbidímetros. Por tanto, se debe tener en cuenta que las estimaciones de concentraciones de sedimento en suspensión están afectadas por un importante margen de incertidumbre.

Asimismo, los registros de turbidez muestran importantes diferencias, que supondrían que la concentración de sedimento en suspensión presenta una elevada variabilidad temporal, con diferencias del pico máximo que pueden ser de hasta 30 veces (entre 4,2 y $\left.1.230 \mathrm{~g} \cdot \mathrm{l}^{-1}\right)$. Al clasificar los eventos según el valor máximo de concentración se observa que: en 39 eventos (49\%) no alcanzó los $100 \mathrm{~g} \cdot \mathrm{l}^{-1}$, en 27 eventos (35\%) se situó entre 100 y $500 \mathrm{~g} \cdot l^{-1}$, en 11 eventos $(14 \%)$ quedó entre 500 y $1.000 \mathrm{~g} \cdot \mathrm{l}^{-1}$ y en 2 eventos $(2,5 \%)$ el pico de concentración superó los $1.000 \mathrm{~g} \cdot \mathrm{l}^{-1}$. En todo caso, otro aspecto que llama la atención sobre estos valores de concentración es que son extremadamente elevados. En este sentido, algunos estudios demuestran que la incertidumbre entre turbidez y concentración se incrementan en relación directa con la masa sólida en suspensión (Regüés et al., 2002) dado que la turbidez es muy sensible a las variaciones de algunas propiedades físicas de las partículas en suspensión (Gibbs, 1994; Gippel, 1995). Sin embargo, también hay algunos indicios que podrían sugerir que estas estimaciones se podrían aproximar a la realidad. Entre estos indicios estarían las concentraciones máximas medidas en las muestras tomadas por el ISCO, que frecuentemente superan los 100 $\mathrm{g} \cdot \mathrm{l}^{-1}$ e incluso han llegado a alcanzar los $200 \mathrm{~g} \cdot \mathrm{l}^{-1}$. Aunque esto se podría interpretar como la demostración de la sobreestimación de las concentraciones también se puede utilizar en sentido contrario, porque se ha observado que estos instrumentos muestran ciertas limitaciones asociadas a su capacidad de succión y no suelen captar partículas mayores de un cierto diámetro (Soler et al., 2003). Esta limitación implicaría que los sistemas de muestreo automático subestiman las concentraciones, como consecuencia del muestreo deficiente de las partículas de mayor tamaño, ya que la relación entre el diámetro de las partículas y su masa es de tipo exponencial, por lo que la ausencia de partículas mayores alteraría notablemente esta estimación. Y, como es lógico, este efecto tiene mayor trascendencia cuando el transporte en suspensión es variable y heterogéneo. Por otro lado, en los estudios sobre transporte de sedimento realizados en la cárcavas de Draix (Mathys et al., 2003) se han registrado valores máximos de concentración de sedimento en suspensión que alcanzan los $800 \mathrm{~g} \cdot \mathrm{l}^{-1}$, lo cual se podría considerar un argumento 
a favor de la validez de los valores de concentración estimados, pero en este caso también se trata de estimaciones a partir de la turbidez y, por tanto, pueden estar afectados por las mismas incertidumbres.

En cualquier caso, con independencia de los errores implícitos en la cuantificación del transporte en suspensión, los datos registrados en la cuenca de Araguás ponen de manifiesto que se trata de un proceso muy variable y heterogéneo. Esto sugiere que se deben tener en cuenta los factores que intervienen en la producción de sedimento y su susceptibilidad a ser movilizado, como el estado físico del regolito en las laderas y la disponibilidad de sedimento, o las características de la precipitación y de la respuesta hidrológica. La influencia de estas variables en el transporte se ha explorado a partir de regresiones lineales, en las que se han considerado los valores medios y máximos de concentración de sedimento en suspensión y la masa de sedimento exportado como variables dependientes y un conjunto de variables que describen las condiciones en que se han producido las crecidas (estado físico del regolito superficial, características de la precipitación y respuesta hidrológica) como variables independientes. Asimismo, se ha tomado como criterio secundario la diferenciación estacional de las crecidas (estación seca y húmeda).

Los resultados (Tabla 2) indican que la cantidad total de sedimento exportado se correlaciona algo mejor con el conjunto de variables que las concentraciones, lo cual es lógico dado que el transporte se obtiene como producto de la concentración por el caudal. Asimismo, se observan diferencias significativas entre los coeficientes de correlación al analizar separadamente el transporte en las estaciones seca y húmeda, reflejando el efecto de la dinámica de las laderas sobre la capacidad de generación de escorrentía $\mathrm{y}$, por tanto, las variaciones temporales de la capacidad de transferencia de sedimento hacia las redes de drenaje (Regüés y Gallart, 2004; Nadal-Romero et al., 2008b y NadalRomero y Regüés, 2009). Así, en estación húmeda la concentración máxima se correlaciona de manera directa con la resistencia mecánica superficial (RMS) y de manera indirecta con la humedad superficial, lo cual pone de manifiesto la influencia que produce el estado físico del regolito superficial sobre la respuesta hidrológica y la producción de sedimento; por un lado cuando presenta una morfología tipo "popcorn" (máxima humedad y mínima RMS) y, por otro lado, cuando se encuentra sellada (humedad superficial alta o moderada y mayor RMS); en el primer caso porque el "popcorn" causa una capacidad de infiltración muy elevada y, por consiguiente, el alcance de la transferencia de sedimento suele estar restringido hasta el pie de las laderas, mientas en el segundo caso la capacidad de infiltración es muy baja y, por consiguiente, la escorrentía en las laderas de las cárcavas es rápida e intensa y genera flujos capaces de erosionar y arrastrar el sedimento. Algo similar se observa durante la estación seca con la relación directa entre la densidad aparente y la exportación total: mientras la densidad aparente se mantiene baja (generalmente al principio de primavera cuando se mantiene todavía la morfología popcorn en las laderas) la erosión por salpicadura es intensa, aunque la capacidad del infiltración continúa siendo elevada y el flujo de escorrentía superficial es limitado, lo que se acumulan grandes cantidades de sedimento al pie de las laderas. Esta situación cambia a medida que se desmorona la morfología popcorn y aumenta la densidad del 


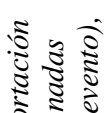

ปั

ว $\frac{2}{2}$

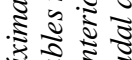

ฐ

हิ

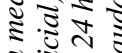

:

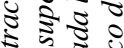

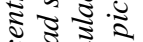

ช

.ิ

ปั

तิ

ล के है

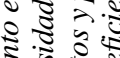

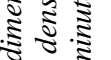

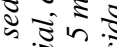

ะ

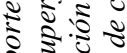

के

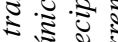

₹

ระ

总胥.

2.

$\frac{8}{2}=\frac{2}{2} \cdot \frac{3}{3}$

는

ये

$\therefore \frac{\bar{\Xi}}{2}$

205

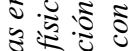

. 8.

ริำ

ป ะ

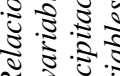

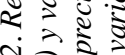

กิ

胥

\begin{tabular}{|c|c|c|c|c|c|c|c|c|c|c|c|c|c|}
\hline \multirow{3}{*}{ 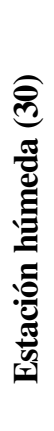 } & 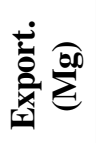 & $\begin{array}{l}\text { * } \\
\text { đ్ర } \\
\text { ర్ } \\
0\end{array}$ & $\begin{array}{l}\infty \\
8 \\
8 \\
0\end{array}$ & ڤ̂. & \multirow{3}{*}{ 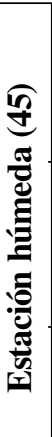 } & 离 & 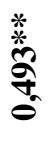 & 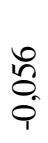 & @ొ & $\begin{array}{l}\infty \\
8 \\
0 \\
0 \\
0\end{array}$ & 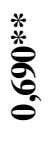 & 葛 & 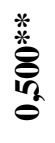 \\
\hline & שֶ & $\begin{array}{l}\frac{*}{*} \\
\frac{*}{n} \\
\tilde{n}^{\prime}\end{array}$ & $\frac{\infty}{2}$ & î & & 己 & กู & $\stackrel{+}{5}$ & $\frac{\stackrel{\infty}{+}}{0_{1}^{+}}$ & $\begin{array}{l}m \\
0 \\
0 \\
0\end{array}$ & $\begin{array}{l}\stackrel{\infty}{0} \\
\text { ஸू }\end{array}$ & $\frac{\infty}{\stackrel{n}{0}}$ & ปู \\
\hline & ڤ્仓 & $\stackrel{m}{2}_{0}^{2}$ & $\begin{array}{l}m \\
\hat{0} \\
0\end{array}$ & है & & $\tilde{\theta}$ & ڤ్ & $\begin{array}{l}\infty \\
8 \\
0 \\
0 \\
0\end{array}$ & $\frac{1}{\equiv}$ & $\begin{array}{l}7 \\
0 \\
0 \\
0\end{array}$ & ฮิ & $\begin{array}{l}0 \\
0 \\
0 \\
0\end{array}$ & $\stackrel{8}{8}$ \\
\hline
\end{tabular}

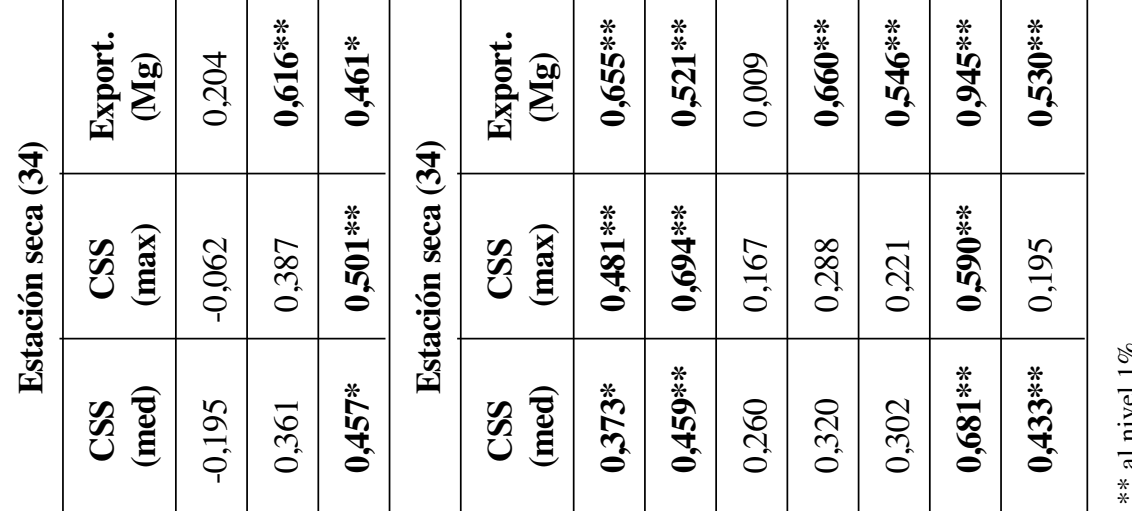

\begin{tabular}{|c|c|c|c|c|c|c|c|c|c|c|c|c|}
\hline 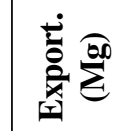 & $\frac{0}{\tilde{n}}$ & $\stackrel{*}{\stackrel{*}{\sigma}}$ & $\begin{array}{l}\infty \\
\stackrel{0}{0} \\
0\end{array}$ & 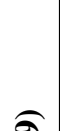 & 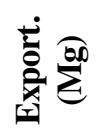 & 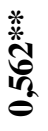 & $\stackrel{*}{\text { กู }}$ & 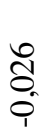 & 葛 & 蒡 & 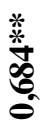 & 草 \\
\hline 己) & ঠ̊. & 广̊ & ֻู & $\begin{array}{l}\text { 总 } \\
\frac{0}{2} \\
=\end{array}$ & ש & 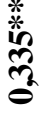 & 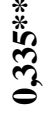 & ڤై & సू. & $\frac{\infty}{n}$ & 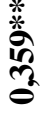 & $\frac{\infty}{n}$ \\
\hline ש્己 & $\begin{array}{l}\infty \\
\infty \\
\stackrel{0}{n} \\
0\end{array}$ & $\frac{1}{\sigma}$ & 葢 & & $\underset{\mathscr{D}}{\tilde{d}}$ & $\frac{\infty}{n}$ & $\begin{array}{l}0 \\
0 \\
0\end{array}$ & $\begin{array}{l}\infty \\
0 \\
0 \\
0\end{array}$ & $\frac{\hat{0}}{0}$ & 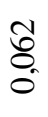 & हू & $\begin{array}{l}\text { तु } \\
\stackrel{0}{0} \\
0\end{array}$ \\
\hline & 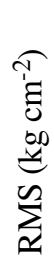 & 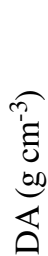 & 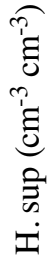 & & & ఏ્ఏ & 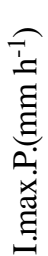 & 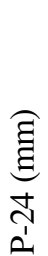 & 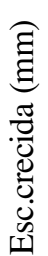 & 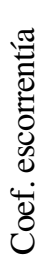 & 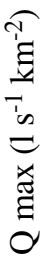 & 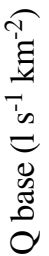 \\
\hline
\end{tabular}


regolito hasta alcanzar valores elevados (generalmente entre el final de primavera y la primera mitad del verano), en estas condiciones se reduce significativamente la capacidad de infiltración y se generan flujos con capacidad de erosionar y arrastrar el sedimento acumulado. Este mismo efecto se puede asociar a la correlación directa entre concentración máxima y exportación de sedimento con la humedad superficial, puesto que es una de las variables que afecta a la capacidad de generación de escorrentía durante la estación seca (Nadal-Romero et al., 2008a).

Por otro lado, también se observan importantes diferencias estacionales en las relaciones entre las concentraciones y las variables asociadas a la precipitación y la respuesta hidrológica (Tabla 2). Así, durante la estación seca la mitad de las variables se correlacionan significativamente, mientras en la estación húmeda ninguna variable se correlaciona significativamente. Esto se puede asociar al desfase temporal que existe entre la máxima disponibilidad de sedimento y la máxima capacidad de generación de caudales, y a las diferencias entre los procesos que causan la respuesta hidrológica en las cárcavas y en las zonas vegetadas. Este desfase temporal causa con frecuencia un efecto de dilución e incluso de cierto agotamiento del sedimento disponible en la estación húmeda, por el aporte de agua "limpia" desde las zonas vegetadas y porque la transferencia de sedimento desde las laderas de las cárcavas hacia el barranco generalmente es bastante más lenta que el ritmo con que se elimina el sedimento acumulado en el cauce. Esto ocasiona que ni las precipitaciones, ni la escorrentía, ni la magnitud del caudal se relacionen con las concentraciones, aunque sí hay una buena relación con la exportación; sin embargo, durante el inicio de la estación seca la disponibilidad de sedimento es muy elevada, pero los caudales están limitados porque la escorrentía procede casi exclusivamente de las laderas de las cárcavas y está muy asociada a la intensidad de precipitación. Además en esta situación se combina el efecto de una mayor eficacia de la erosión por salpicadura, que se pone de manifiesto a través de la buena correlación $(\mathrm{R}=0,694)$ entre la intensidad de precipitación y las concentraciones (Tabla 2), y la eficacia de la transferencia por arroyada superficial, que se evidencia en la correlación significativa $(0,66)$ entre el volumen de escorrentía durante las crecidas y la exportación total (Tabla 2). Estos factores contribuyen a que los caudales llevan generalmente una carga de sedimento mucho más constante que en la estación húmeda, que se evidencia a través de la mayor proporcionalidad entre la magnitud del caudal (Qmax) y las concentraciones (CSSmed,max) o el transporte total (Export) (Tabla 2).

\subsubsection{El transporte a escala anual}

La cuantificación del transporte de sedimento en suspensión se ha evaluado a partir de los valores de concentraciones y caudales registrados en la estación de aforo. Estos datos proceden de un solo año hidrológico (2005-2006) y, por tanto, no se ha podido valorar en un contexto temporal más amplio la representatividad de la tasa estimada. Así, el valor absoluto de la masa de sedimento exportado en suspensión estimado para este periodo de 12 meses oscilaría entre un mínimo de 1.400 y un máximo de $6.900 \mathrm{Mg}$. Este intervalo se ha obtenido aplicando un rango de incertidumbre para las medidas de con- 
centración de sedimento en suspensión de medio orden de magnitud, que corresponde al factor de proporcionalidad medio más frecuente entre los valores estimados a partir del turbidímetro y las medidas de concentraciones adquiridas por el ISCO durante las crecidas. Así, la tasa de exportación de sedimento se situaría entre 3.067 y $15.333 \mathrm{Mg} \mathrm{km}^{-2}$ año ${ }^{-1}$ si se considera la superficie total de la cuenca $\left(0,45 \mathrm{~km}^{2}\right)$, o $11.500-57.500 \mathrm{Mg} \mathrm{km}^{-2}$ $a_{\tilde{n}} \mathrm{o}^{-1}$ si se relaciona únicamente con la superficie acarcavada $\left(0,12 \mathrm{~km}^{2}\right)$. Estas tasas de exportación de sedimento se pueden aplicar a los $15 \mathrm{~km}^{2}$ de cárcavas desarrolladas en de la Depresión Interior Altoaragonesa, en cuyo caso se obtendría que la cantidad total de sedimento incorporado a las redes de drenaje durante el año hidrológico 2005-06 podría oscilar entre los 46.000 y $230.000 \mathrm{Mg}$, lo que representaría un volumen de sedimento de entre 37.000 y $184.000 \mathrm{~m}^{3}$ tomando un valor de densidad aparente de $1,25 \mathrm{~g} \cdot \mathrm{cm}^{-3}$. Si se contrastan estas cifras con la capacidad del embalse de Yesa (alrededor de $470 \mathrm{hm}^{3}$ ) se llega a la conclusión de que estas cantidades de sedimento representan un volumen que causarían una reducción anual del 0,04 \% de su volumen y, por tanto, estas magnitudes de transporte de sedimento no deberían representar una amenaza.

Por otro lado, el análisis de los datos obtenidos en los 6 meses siguientes (octubre 2006-abril 2007) indicarían un transporte de entre 230 y $1.150 \mathrm{Mg}$, que representarían un producción media de entre 510 y $2.550 \mathrm{Mg} \mathrm{km}^{-2}$ año-1 para toda la cuenca o entre 1.960 y $9.800 \mathrm{Mg} \mathrm{km}^{-2}$ año $^{-1}$ en la superficie total de las cárcavas. Sin embargo, una estimación obtenida a partir de 6 meses de datos no puede ser valorada como una tasa anual y, menos aún, contrastarla con un valor obtenido a partir del registro de un año hidrológico completo. Por desgracia los registros de datos sedimentológicos en la estación de aforo quedaron interrumpidos por causa del robo de instrumentos y los destrozos que sufrió la estación de aforo en abril de 2007. En todo caso, se observa que ambas tasas de exportación no difieren excesivamente $\mathrm{y}$, por tanto se puede extraer de estas primeras aproximaciones que la magnitud del transporte en suspensión puede oscilar entre un factor de $10^{3}$ hasta uno de $10^{5} \mathrm{Mg} \mathrm{km}^{-2}$ año ${ }^{-1}$ en las cárcavas, que es un rango similar al observado en las cárcavas de montaña de "El Carot" y "Draix" (Regüés et al., 2000a; Mathys et al., 2003) pero muy superior a los estimados en ambientes menos degradados (García-Ruiz et al., 2008).

Asimismo, se pueden extraer algunas conclusiones sobre la representatividad de esta tasa en un contexto temporal más amplio, mediante la comparación de los registros de precipitación obtenidos en la cuenca de Araguás con otras series de datos obtenidos en el Pirineo Central. Así, la precipitación media anual registrada entre 2006 y 2007 en la cuenca de Araguás es de 666,5 mm, siendo 2006 el año más lluvioso con 702 mm, mientras en la localidad de Castiello de Jaca la precipitación media en este trienio alcanzó los 790 mm y en 2006 los 976,7 mm Esta información demuestra la importante variabilidad espacial que afecta al Pirineo Central, pero también que hay importantes diferencias temporales. Esta variabilidad interanual es mucho más evidente al considerar una serie de datos más larga: la precipitación anual recogida en Castiello de Jaca entre 1968 y 2006 (Fig. 4) indica que el valor media anual se situaría alrededor de $1015 \mathrm{~mm}$ y una gran variabilidad entre el año más lluvioso $(1539,2 \mathrm{~mm})$ y el más seco $(631,6 \mathrm{~mm})$. Así, la estimación de exportación de sedimento en la cuenca de Araguás se ha realizado en 


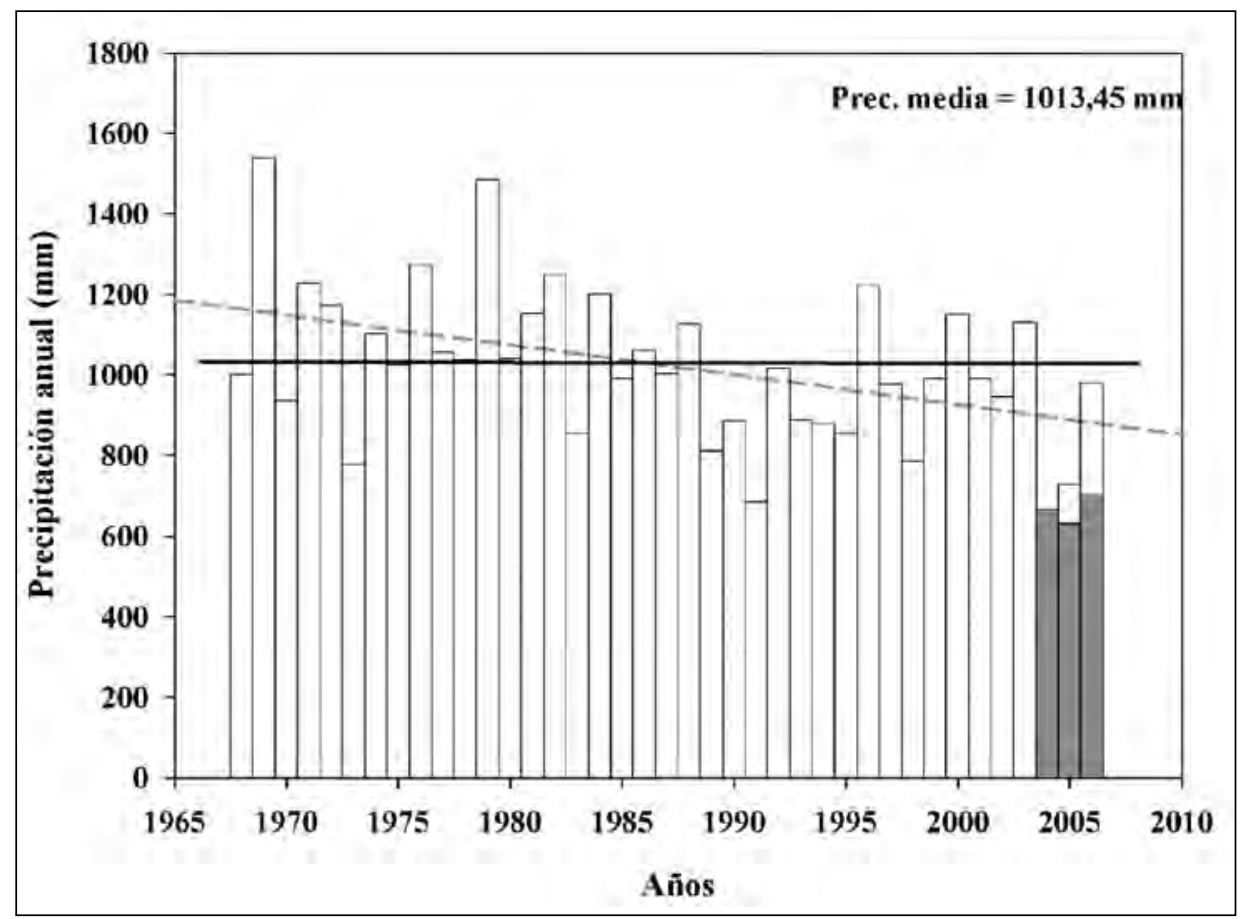

Figura 4. Registros de precipitación anual correspondientes a Castiello de Jaca (1968-2006) y la cuenca de Araguás (2004-2006)

un periodo con precipitación por debajo de la media y, por consiguiente, la respuesta hidro-sedimentológica posiblemente se ha situado en valores entre bajos y moderados. Esta hipótesis queda avalada mediante las relaciones estadísticas analizadas anteriormente, entre las que se puede destacar la buena asociación que existe entre las concentraciones de sedimento y las intensidades y cantidades de precipitación, especialmente durante las estaciones más secas, o también la buena relación que existe entre la exportación de sedimento y los caudales, que también dependen fuertemente de las precipitaciones (Nadal-Romero et al., 2008a).

Por otro lado, el evento pluviométrico de mayor envergadura registrado en la cuenca de Araguás alcanzó los 49,8 mm (23 de septiembre de 2006) y corresponde a un periodo de retorno de 1,8 años (García-Ruíz el al., 2000). Esto quiere decir que este evento es habitual en esta zona y su efecto hidro-sedimentológico no puede considerarse excepcional. En este sentido, se ha demostrado que la relación entre el transporte de sedimento y la cantidad de precipitación no es proporcional (Edwards y Owens, 1991; Regüés et al., 2000a), de manera que las consecuencias hidro-sedimentológicas asociadas a las precipitaciones de mayor magnitud (con periodos de recurrencia más largos) son equivalentes a las producidas por muchos eventos menores acumulados. La Figura 5 


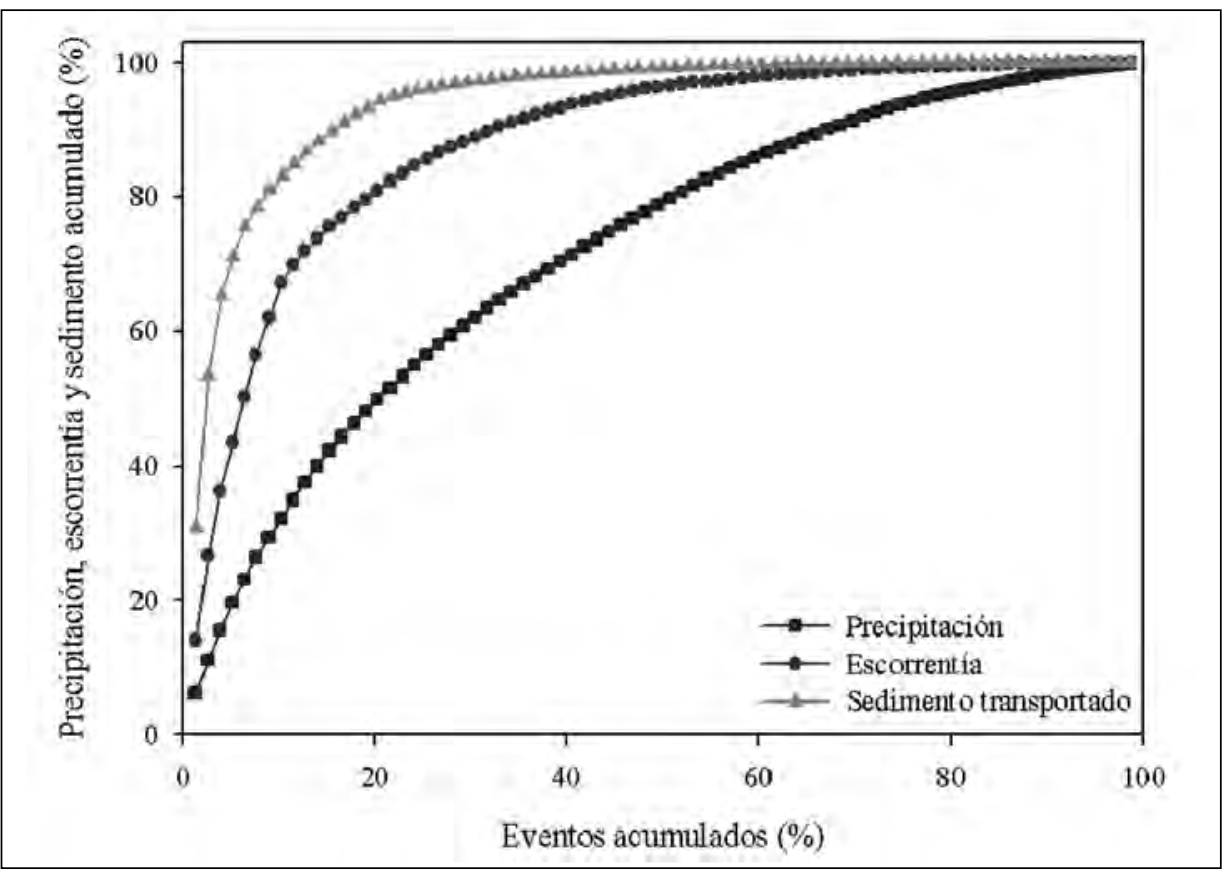

Figura 5. Relación entre los porcentajes de sedimento en suspensión exportado y los de precipitación y escorrentía. Los datos están referidos al porcentaje de eventos en orden inverso a la magnitud del transporte asociado a ellos

ilustra el gran impacto que producen las precipitaciones de mayor magnitud en la respuesta hidrológica y el transporte de sedimento en la cuenca de Araguás, reflejada a través de las diferencias existentes en las pendientes de las curvas acumulativas que presentan el sedimento y la escorrentía con respecto a la precipitación asociada. Estas diferencias muestran que un gran porcentaje del transporte y la escorrentía está relacionado con unos pocos eventos que, aún siendo los de mayor magnitud, representan un porcentaje relativamente pequeño de la precipitación total anual.

\subsubsection{Distribución temporal del transporte de sedimento en suspensión}

Los resultados obtenidos en la cuenca de Araguás muestran que la dinámica geomorfológica en las cárcavas de montaña se caracteriza por su gran sensibilidad frente a las variaciones de las condiciones climáticas estacionales, lo cual se refleja en una compleja secuencia de procesos y respuestas. Así, la intensidad y eficacia que muestran los procesos de meteorización física y erosión están directamente reflejadas en la intensidad y eficacia de la respuesta hidrológica y en la producción de sedimento en las laderas de las cárcavas (Regüés y Gallart, 2004; Nadal-Romero y Regüés, 2009). 
La veracidad de estas afirmaciones sobre las diferencias existentes entre las relaciones causa-efecto, en relación con la exportación de sedimento, precisa analizar la información disponible en una determinada escala temporal. Este objetivo no se podría alcanzar a partir de un análisis individualizado de las crecidas, puesto que cada caso presenta una variedad de matices excesivamente amplia, pero se puede obtener a partir de considerar unas condiciones generalizables tanto para la superficie de las cárcavas como para las condiciones climáticas. Esto implica que la escala temporal más detallada que se puede utilizar para este análisis sea la mensual y la más amplia la estacional.

La Figura 6 ilustra la distribución mensual de las producciones de sedimento, la escorrentía y la precipitación acumuladas en la cuenca de Araguás. En este caso se pueden observar algunos aspectos interesantes relacionados con la distribución temporal del transporte. En primer lugar, se pone de manifiesto que la exportación del sedimento en suspensión se produce principalmente a finales de invierno y en los primeros meses del otoño, coincidiendo con los dos momentos de mayor producción de escorrentía. En segundo lugar, se puede destacar que el desfase temporal entre los procesos de meteorización, erosión y transporte origina una cierta limitación en la producción de sedimento, esto se pone de manifiesto en las consecuencias derivadas de la gran producción de sedimento en el mes de marzo de 2006, que no mantiene la misma intensidad ni en septiembre ni durante el resto del otoño, a pesar de ser el periodo de mayor producción de escorrentía. Estas observaciones demuestran cierta variabilidad en el efecto hidro-sedimentológico de las precipitaciones, dependiendo del estado físico de las superficie de las cárcavas y de la acumulación de sedimento en el pie de las vertientes. Este hecho también se pone de manifiesto a través de la desproporción que se observa entre las cantidades totales de sedimento y la precipitación acumulada, algo que no sucede con el volumen de escorrentía, puesto que es más proporcional a la cantidad total de precipitación.

La Figura 7 expone la distribución estacional de las cantidades totales acumuladas de sedimento, escorrentía y precipitación. Lo más destacable en este caso son las importantes diferencias asociadas a la producción de sedimento, que no parecen depender ni de la precipitación ni de la escorrentía acumuladas. Sin embargo, se observa que la producción de escorrentía está bastante bien relacionada con la precipitación total, con independencia de la magnitud del evento o de las condiciones climáticas (Nadal-Romero, 2008). Esto sugiere que las cárcavas deberían ser consideradas principalmente como áreas fuente de escorrentía, incluso por encima de su evidente protagonismo en la producción de sedimento, puesto que la producción de sedimento es mucho más variable y dependen principalmente de la intensidad y eficacia con que actúan los procesos de meteorización física sobre la roca margosa. Finalmente, la producción de escorrentía está limitada exclusivamente por las precipitaciones, de manera que el rápido incremento de las temperaturas que se viene registrando en los últimos años, y se encuentra entre las principales causas-efectos del cambio climático, podría causar una reducción de la producción de sedimento si disminuye la meteorización física asociada al crecimiento de hielo, pero no debería afectar de manera significativa a la producción de escorrentía en las cárcavas si no se producen variaciones significativas en las cantidades totales de precipitación. 


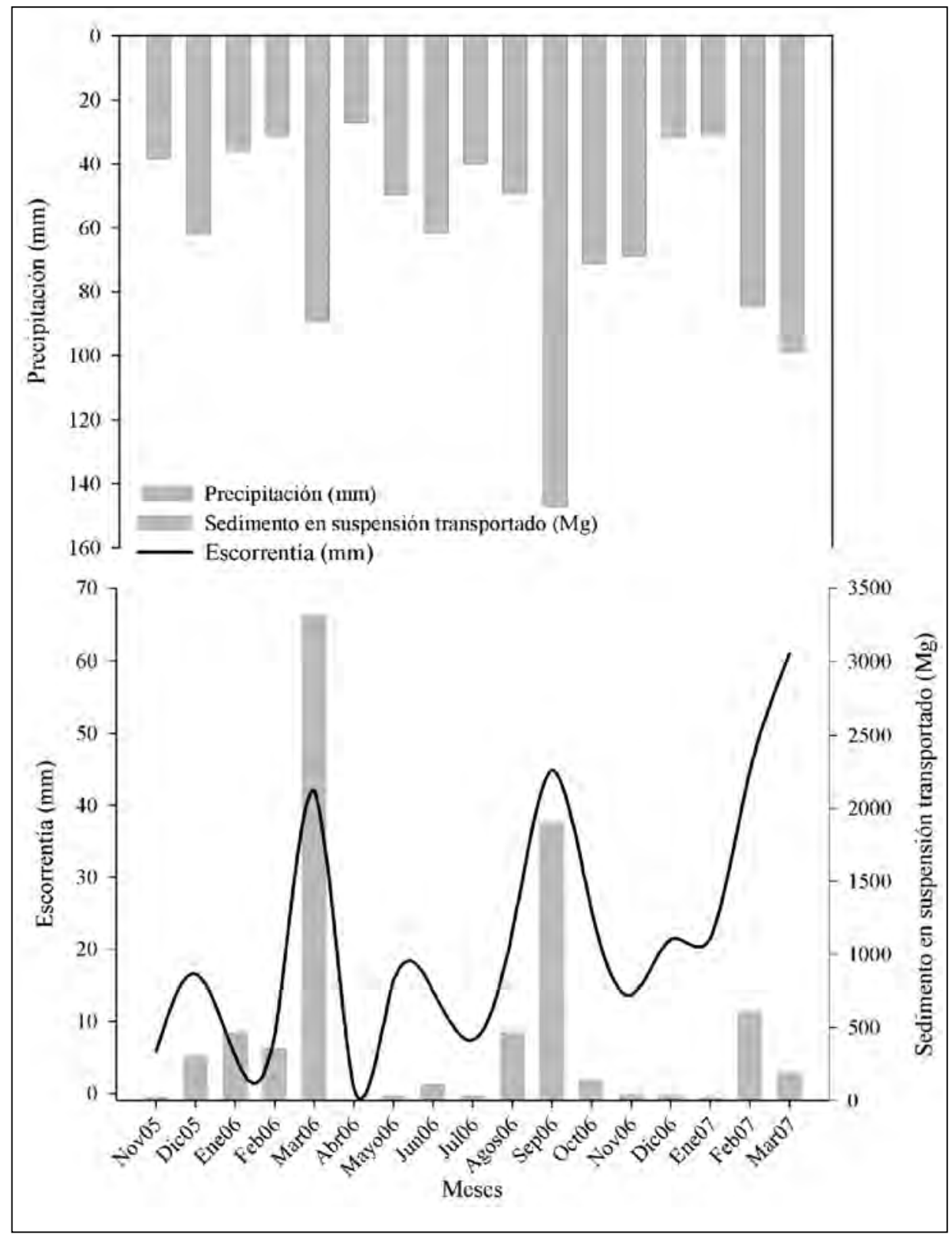

Figura 6. Distribución mensual de las cantidades acumuladas de precipitación, escorrentía y transporte de sedimento en suspensión 


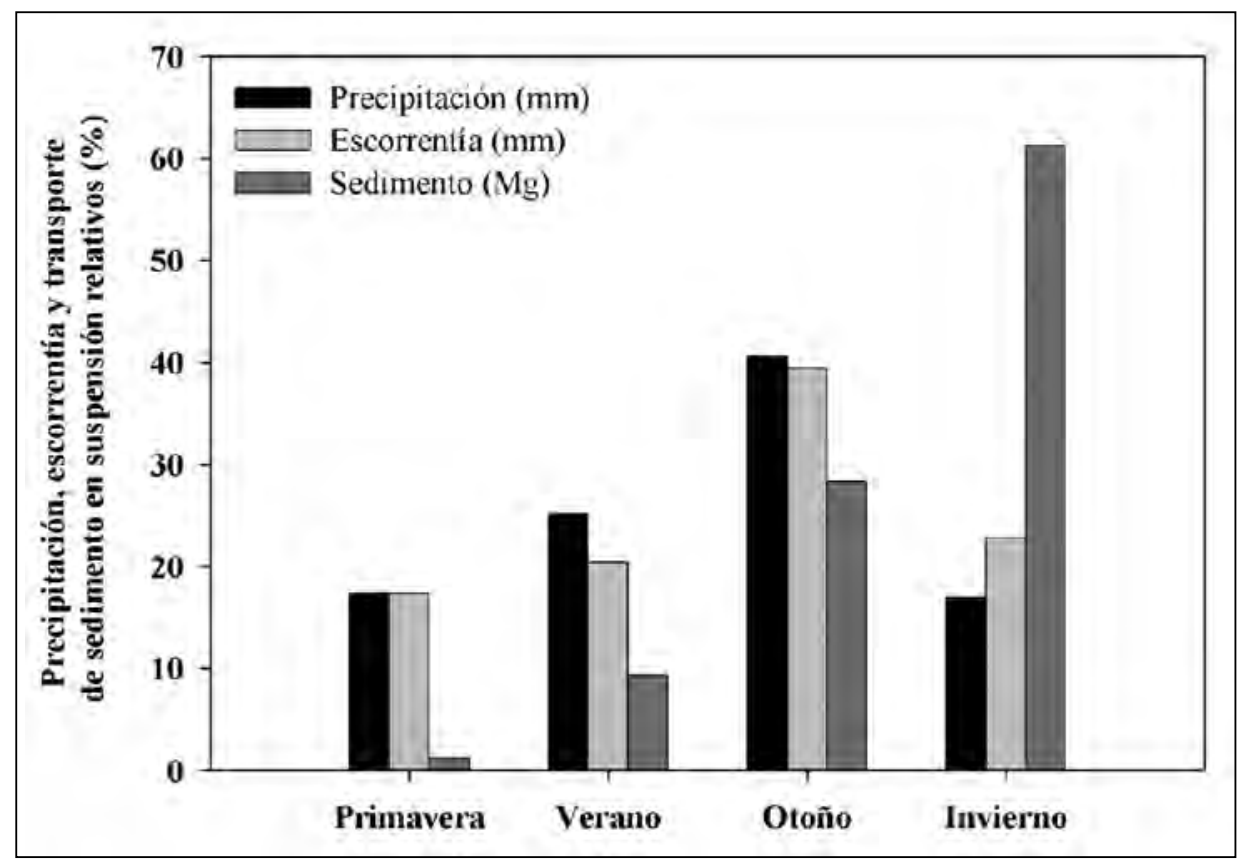

Figura 7. Producción estacional de escorrentía y sedimento en la cuenca de Araguás

\section{Conclusiones}

La producción de sedimento en las cárcavas desarrolladas en la Depresión Interior Altoaragonesa sugiere claramente que está regulada por una secuencia estacional de procesos. Esta dinámica temporal depende principalmente de la intensidad con que actúan los procesos de meteorización física, asociada a la acción del hielo-deshielo principalmente, y de erosión, por la gran eficacia de la salpicadura asociada a tormentas de fuerte intensidad. Estos factores determinan fuertemente la disponibilidad de material susceptible a ser arrastrado por la escorrentía, y los registros de sedimento en suspensión y caudales demuestran que el aporte de sedimento desde estas morfologías disminuye de manera notable durante los periodos más cálidos. Estas fluctuaciones tan claras parecen afectar mucho menos a la producción de escorrentía, lo cual puede indicar que el rápido incremento de las temperaturas en el presente contexto de cambio climático debería afectar más la producción de sedimento que al volumen de agua en estos ambientes. Sin embargo, estas conclusiones son hipótesis formuladas a partir de una serie de datos excesivamente corta y, por ello, se deben considerar como tales. La ampliación del registro de datos en el futuro permitirá realizar un análisis de la información más riguroso y consistente. 


\section{Agradecimientos}

Este trabajo se ha realizado con el apoyo de los siguientes proyectos: "Procesos y balances de sedimentos a diferentes escalas espaciales en ambientes mediterráneos: Efectos de las fluctuaciones climáticas y los cambios de uso del suelo" (PROBASE, CGL2006-11619/HID) y "Comportamiento y modelización espacio temporal de la transferencia de sedimentos en distintos usos del suelo" (CETSUS, CGL2007-66644C04-01/HID) todos financiados por la CICYT, y "Validación de un modelo acoplado de simulación de procesos hidrológicos e hidráulicos utilizando datos de cuencas experimentales en montaña mediterránea" (PM088/2006), financiado por la Diputación General de Aragón (DGA). El mantenimiento y mejora de la cuenca se ha llevado a cabo mediante el Convenio CSIC/Ministerio de Agricultura (Dirección General de Conservación de la Naturaleza) - "Red de estaciones Experimentales de seguimiento y evaluación de la erosión y desertificación" (RESEL), financiado por el Ministerio de Agricultura. El segundo autor contó con una beca predoctoral I3P concedida por el CSIC en el contexto del Programa Social Europeo.

\section{Referencias bibliográficas}

AleXandrov, Y., LaRonne, J.B., ReID, I. (2003). Suspended sediment concentration and its variation with water discharge in dryland ephemeral channel, northern Negev, Israel. Journal of Arid Environments, 53: 73-84.

Antoine, P., Giraud, D., Meunier, M., VAN Ash, T. (1995). Geological and geotechnical properties of the "Terres Noires" in southeastern France: weathering, erosion, solid transport and instability. Engineering Geology, 40: 223-234.

BEGUERÍA, S. (2005). Erosión y fuentes de sedimento en la cuenca del embalse de yesa (Pirineo Occidental): Ensayo de una metodología basada en teledetección y análisis de SIG. Tesis Doctoral, Instituto Pirenaico de Ecología (CSIC). 158 pp. Zaragoza.

Beguería, S., Vicente-SERRANO, S.M. (2006). Mapping the hazard of extreme rainfall by peaks over threshold extreme value analysis and spatial regression techniques. Journal of Applied Meteorology and Climatology, 45 (1): 108-124.

BRYAN, R.B., YAIR, A. (1982). Perspectives on studies of badland geomorphology. En: Badland Geomorphology and Piping. Geobooks, pp. 1-12, Norwich.

Calvo-Cases, A., Harvey, A.M. (1996). Morphology and development of selected badlands in Southeastern Spain: implications on climate change. Earth Surface Processes and Landforms, 21: 725-735.

CAMPBELl, B.E., SPINRAD, R.W. (1987). The relationship between light attenuation and particle characteristics in a turbid estuary. Estuar.Coast.Mar.Sci., 25: 53-65. 
Cantón, Y., Domingo, F., Solé-Benet, A., Puigdefábregas, J. (2001). Hydrological and erosion response of a badlands system in semiarid SE Spain. Journal of Hydrology, 252 (9): 65-84.

Cerdá, A., NAVArro, R. (1997). Procesos de erosión en los badlands alicantinos. Investigaciones geográficas, 17: 99-116.

Creus, J. (1983). El clima del Alto Aragón Occidental. Monografías del Instituto de Estudios Pirenaicos, 109, 233 pp. Jaca.

EDWARDS, W.M., OWENS, L.B. (1991). Large storms effects on total soil erosion. Journal of Soil and Water Conservation, 46: 75-78.

Gallart, F., SolÉ, A., Puigdefábregas, J., LÁZARo, R. (2002). Badland systems in the Mediterranean. En: Dryland Rivers: Hydrology and Geomorphology of Semi-arid Channels (Bull, J.L., Kirkby, M.J., Eds.). John Wiley \& Sons, Ltd. pp. 299-326. Chichester.

GarcíA-Ruiz, J.M., ARnÁEZ, J., White, S., Lorente, A., BegueríA, S. (2000). Uncertainty assessment in the prediction of extreme rainfall events: an example from the central Spanish Pyrenees. Hydrological Processes, 14: 887-898.

García-Ruiz, J.M., RegüÉs, D., Alvera, B., LANA-RenAult, N., SERRANO-MuEla, P., Nadal-Romero, E., Navas, A., Latron, J., Martí-Bono, C., Arnáez., J. (2008). Flood generation and sediment transport in experimental catchments affected by land use changes in the central Pyrenees. Journal of Hydrology, 356: 245-260.

GIBBS, R.J. (1994). Principles of studying suspended material in water. En: Suspended Solids in Water (Gibbs, R.J., Eds.), Plenum Press, pp. 3-16, New York.

GIPPEL, CH.J. (1995). Potential of turbidity monitoring for measuring the transport of suspended solids in streams. Hydrological Processes, 9: 83-97.

HAIGH, M.J. (1978). Microrills and desiccation cracks: Some observations. Z. Geomorphology, 22(4): 457-461.

LÓPEZ-BERMúdez, F., RoMERO-DÍAZ, M.A. (1989). Piping erosion and badland development in south-east Spain. Catena Suppl.-Bd., 14: 59-73.

MCINTYRE, D.D. (1958). Soil splash and the formation of surface crust by raindrop impact. Soil Science, 85: 261-266.

Martí-Bono, C., Plana, F. (1996). Concreciones carbonatadas en margas del Eoceno. Consecuencias geomorfológicas. En: Cadernos do Laboratorio Xeolóxico de Laxe (Grandal, A., Pagés, L., Eds.), pp. 604-615. Santiago de Compostela.

MATHYS, N., RICHARD, D., GRÉSILLON, J.M. (2003). Non-linearity in erosion response of a small mountainous and marly basin: The Laval in the Draix experimental catchments, South East, France. Int. Conf. Hydrology of the Mediterranean and semi-arid regions, Montpellier, France. CD-ROM. 
Mathys, N., Klotz, S., Esteves, M., DescroiX, L., Lapetite, J.M. (2005). Runoff and erosion in the Black Marls of the French Alps: observations and measurements at the plot scale. Catena, 63: 261-281.

Nadal-Romero, E., Regües, D., Lana-Renault, N., Serrano-Muela, P., MartíBONO, C. (2006a). Impacto hidrológico y sedimentológico de las morfologías acarcavadas en la depresión interior altoaragonés (Pirineo Central). En: Geomorfología y Territorio (Pérez Alberti; A., López Bedoya, J.) vol. 171, pp. 615-628.

Nadal-Romero, E., Regǘés, D., Martí-Bono, C., Serrano-Muela, P. (2006b). Dinámica estacional de los procesos de meteorización en cárcavas del Pirineo Central. Cuaternario y Geomorfología, 20 (1-2): 61-77.

Nadal-Romero, E., Regüés, D., Martí-Bono, C., Serrano-Muela, P. (2007). Badlands dynamics in the Central Pyrenees: temporal and spatial patterns of weathering processes. Earth Surface Processes and Landforms, 32(6): 888-904.

NADAL-Romero, E. (2008). Las áreas de cárcavas (badlands) como fuente de sedimento en cuencas de montaña: procesos de erosión, meteorización, erosión y transporte en margas del Pirineo Central. Tesis Doctoral inédita. Universidad de Zaragoza, 434 pp.

Nadal-Romero, E., Latron, J., Lana-Renault, N., Serrano-Muela, P., MartíBONO, C., REGÜÉS, D. (2008a). Temporal variability in hydrological response within a small catchment with badland areas, Central Pyrenees. Hydrological Science Journal, 53 (3): 629-639.

NAdAl-Romero, E., LATron, J., MARTí-Bono, C., RegüÉs, D. (2008b). Temporal distribution of suspended sediment transport in a humid Mediterranean badland area: The Araguás catchment, Central Pyrenees. Geomorphology, 97 (3-4): 601-616. DOI: 10.1016/j.geomorph.2007.09.009.

NADAl-Romero, E., REGÜÉs, D. (2009). Conditioning of detachment by regolith development in a badland system. Earth Surface Processes and Landforms, 34 (6): 824-838.

PARdini, G., VignA-Guidi, G., Pini, R., REgÜÉS, D., GALlART, F. (1996). Structure and porosity of smectitic mudrock as affected by experimental wetting-drying cycles and freezing-thawing cycles. Catena, 27: 149-165.

REgÜÉS, D., PARDinI, G., GALlART, F. (1995). Regolith behaviour and physical weathering of clayey mudrock as dependent on seasonal weather conditions in a badland area at Vallcebre, Eastern Pyrenees. Catena, 25 (1-4): 199-212.

Regǘ́s, D., Balach, J.C., Castelltort, X., Soler, M., Gallart, F. (2000a). Relación entre las tendencias temporales de producción y transporte de sedimentos y las condiciones climáticas en una pequeña cuenca de montaña mediterránea (Vallcebre, Pirineos Orientales). Cuadernos de Investigación Geográfica, 26: 41-65. 
REGÜÉS, D., GUÀRDiA, R., GALlART, F. (2000b). Geomorphic agents versus vegetation spreading as causes of badland occurrence in a Mediterranean subhumid mountainous area. Catena, 40: 173-187.

REGÜÉS, D., TORRI, D. (2002). Efecto de la energía cinética de la lluvia sobre la dinámica de las propiedades físicas y el encostramiento en un suelo arcilloso sin vegetación. Cuaternario y Geomorfología, 16 (1-4): 57-71.

RegüÉs, D., Soler, M., Gallart, F. (2002). Influencia del tamaño de las partículas sobre la estimación de concentraciones de sedimentos en sus pensión mediante turbidímetros de retro-dispersión. En: Estudios recientes (2000-2002) en Geomorfología. Patrimonio, montaña y dinámica territorial. (Serrano, E., García de Celis, A., Guerra, J.C., Morales, C.G., Ortega, M.T., Eds.). Dpto. Geografía-UVA, pp. 239-251.

REGÜÉS, D., GALlaRT, F. (2004). Seasonal patterns of runoff and erosion responses to simulated rainfall in a badland area in Mediterranean mountain conditions (Vallcebre, southeastern Pyrenees). Earth Surface Processes and Landforms, 29: 755-767.

Regüés, D., Alvera, B., lana-Renault, N., Latron, J., Serrano-Muela, P., NAdAl-Romero, E., Martí-Bono, C., GARCÍA-Ruiz, J.M. (2006). Procesos hidrológicos y geomorfológicos en un gradiente de cuencas experimentales. En: Geomorfología y Territorio (Actas de la IX Reunión Nacional de Geomorfología), (Pérez Alberti, A., López Bedoya, J. Eds.), Santiago de Compostela. nº 171, pp. 683-695.

Solé-Benet, A., Calvo, A., Cerdà, A., Lázaro, R., Pini, R., Barberó, J. (1997), Influence of mircro-relief patterns and plant cover on runoff related to processes in Badlands from Tabernas (SE Spain). Catena, 31: 28-38.

SOlER, M., RegÜÉS, D., GAllart, F. (2003). Estudio del tamaño de las partículas en suspensión en relación con el caudal y la concentración de sedimento en una cuenca de montaña. Cuaternario y Geomorfología, 17 (3-4): 69-77.

SOLER, M., GALlART, F. (2006). Diámetro de las partículas transportadas en suspensión en la cuenca de Vallcebre (Pirineos Orientales). En: Geomorfología y Territorio (Pérez Alberti, A., López Bedoya, J. Eds.), Santiago de Compostela. nº 171, pp. 605610.

TORRI, D., RODOLFI, G. (2000). Badlands in changing environments: an introduction. Catena, 40: 119-125.

WEBB, B.W., FOSTER, I.D.L., GuRNELL, A.M. (1995). Hydrolgy, water quality and sediment behaviour. En: Sediment and Water Quality in River Catchments (Foster, I., Gurnell, A., Webb, B., Eds.), John Wiley \& Sons Ltd. pp. 1-30, Chichester.

YAIR, A., LAVEE, H., BRYANN, R.B., ADAR, E. (1980). Runoff and erosion processes and rates in the Zin valley Badlands, Northern Negev, Israel. Earth Surface Processes and Landforms, 5: 205-225. 